\title{
Segmentation of Bony Structures with Ligament Attachment Sites
}

\author{
Heiko Seim ${ }^{1}$, Hans Lamecker ${ }^{1}$, Markus Heller ${ }^{2}$, Stefan Zachow ${ }^{1}$ \\ ${ }^{1}$ Visualisierung und Datenanalyse, Zuse-Institut Berlin (ZIB), 14195 Berlin \\ ${ }^{2}$ Centrum für Muskuloskeletale Chirurgie, Charité-Berlin, 13353 Berlin \\ seim@zib.de
}

\begin{abstract}
This work presents an approach towards reconstructing ligament and tendon attachment sites from 3D medical image data. We apply statistical shape models with an additional free form step to reconstruct the anatomical shape of the distal femur from CT data. After the shape fitting process the locations of ligament attachment sites, which are incorporated in the geometric model, are extracted and their positions optimized via an image based approach. To show the potential of our method we manually extracted the surface of 11 distal femora and their corresponding ligament attachment sites in clinical CT data and compared them to the results of our method.
\end{abstract}

\section{Introduction}

Pre-operative functional simulations of the human lower limbs promise, due to optimized surgery parameters, a reduced number of follow-up surgeries and improved functionality in case of total knee arthroplasty. The prerequisites for such simulations are patient individual geometric models of the anatomy of interest reconstructed from medical image data (i.e. CT, MRI), including for example bone and muscle geometry, muscle centerlines or ligament and tendon attachment sites [1]. The latter ones play an important role in computing the transfer of forces during the simulation. In today's biomechanical models those attachment sites are mostly reduced to points in 3D space, which is a strong simplification, because surface like structures have to be assumed to model forces properly. To overcome this problem Kaptein et al. [2] to our knowledge were the first who presented an approach suited for attachment site reconstruction: geometric models of the human shoulder bones with assigned contours of muscle insertions were fitted to surface models of unknown subjects to predict attachments sites. The method is purely based on geometry information.

\section{Materials and Methods}

By using deformable model segmentation as presented in $[3,4]$ as initialization, our work extends the idea of Kaptein et al. and introduces an additional attachment site fitting step, based on local image features of individual patient's data. 
Fig. 1. Statistical shape model of the knee with outlined attachments sites (left). Shape model fitted to patient data including lateral collateral ligament insertion area (right)
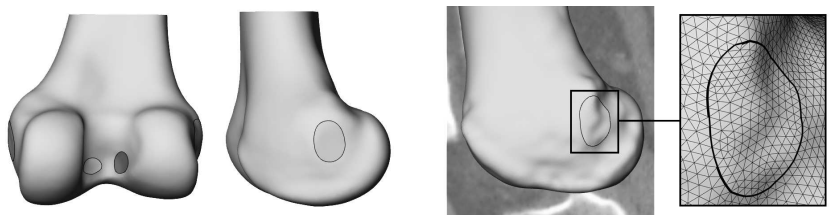

\subsection{Deformable Model Generation and Fitting}

For the creation of the statistical shape model 11 clinical CT data sets of the distal femur with no or very few pathological changes to the bone structure were manually segmented and their surfaces reconstructed. With the assistance of a radiologist we identified the attachment sites of the collateral (MCL, LCL) and cruciate ligaments (PCL, ACL).

Following the approach by [3] a statistical shape model of the distal femur was generated including direct point-to-point correspondences for the attachment sites of all subjects. The result of this process is a statistical atlas describing the average shape of our training set and its geometric variation as well as the location of the included attachment sites (Fig.1).

The alignment of the shape model to CT data follows the idea presented in [4]: First the statistical shape is fitted by adjusting the available degrees of freedom (transformation, rotation, uniform scaling and statistical shape modes). To overcome the restrictive character of statistical shape models, we add a second free-form step: surface vertices are allowed to move in the direction of strong gradients in a user-defined narrow band around the result of the first step.

\subsection{Attachment Site Adaptation}

With the geometric model as initialization (Fig. 2), the attachment site will now be moved to a more patient specific position, analyzing the patient's image data. In CT data of the knee ligaments and tendons are hard to distinguish from

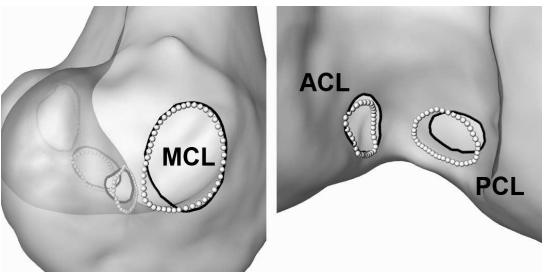

(a)

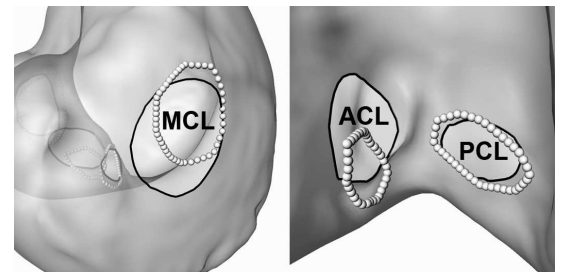

(b)

Fig. 2. Manually (black contour) and geometrically reconstructed attachment sites (spheres). Whereas statistical shape reconstruction often provides good initializations (a), patient individual variations may lead to larger spatial deviations (b) 
other soft tissues structures, even for a trained radiologist. However, there exist certain characteristics that indicate the presence of such structures. Two features seem promising in CT data: Collagen typical intensity values that can be found on the outside of the bone for incoming ligaments (Fig. 3(a)) and areas with increased thickness and density of cortical bone (Fig. 3(b)) created by strong forces working on the bone surface.

By mapping those features onto the bone surface, the problem of finding a patient specific attachment site can be regarded as a $2 \mathrm{D}$ segmentation problem: collagen as well as dense bone tissue create regions of relatively homogeneous intensity on the surface, which can be distinguished from their neighboring structures by intensity differences. The goal is now to move the initial attachment site's contour that comes from the statistical shape model on the surface to match the described image features.

As a first approximate solution to the above mentioned problem and a feasibility study our approach is to tangentially move the initialized contour of the attachment site in a local neighborhood on the extracted bone surface by a globally applied displacement vector $\vec{v}$. This way we are going to suppress unwanted local shape variations caused by prominent but unwanted image features (i.e. very high bone densities at the femoral shaft and notch). We expect the result to be closer to the patient's attachment site than the geometrically initialized contour.

The practical approach is composed of the following steps: First we sample the gray value data along the surface normal of the evenly distributed triangle vertices of the initialized attachment sites. Only the median intensity value along a profile is projected to suppress noise in the CT data. This results in a scalar field on the surface nodes. We found a sampling with a range of $3 \mathrm{~mm}$ inwards for the cruciate ligaments (bone density feature) and 3mm outwards for the PCL (collagen feature) suitable for extracting distinct regions fulfilling the above mentioned criteria. The ACL gave no clear results for both methods, because of the general high bone density in its attachment region and its very acute attachment angle. Considering only the nodes on the initialized attachment sites the following assumption holds for the two extracted features: Nodes with higher

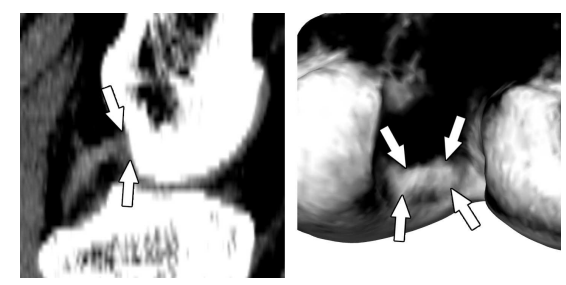

(a)
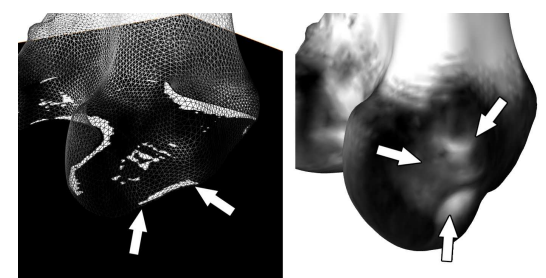

(b)

Fig. 3. Intensity values mapped on the bone surface that indicate an attachment site: Projection of the surrounding soft tissue onto the bone surface creates areas with increased intensity for the PCL (a). By sampling inwards, areas of increased bone density become visible on the surface scalar field (b) 
Fig. 4. Displacement of contour: A displacement vector is computed by evaluating the projected scalar values on every vertex (left). This vector is applied to every contour control point and tangentially projected onto the surface (middle), which yields the new adapted contour (right)
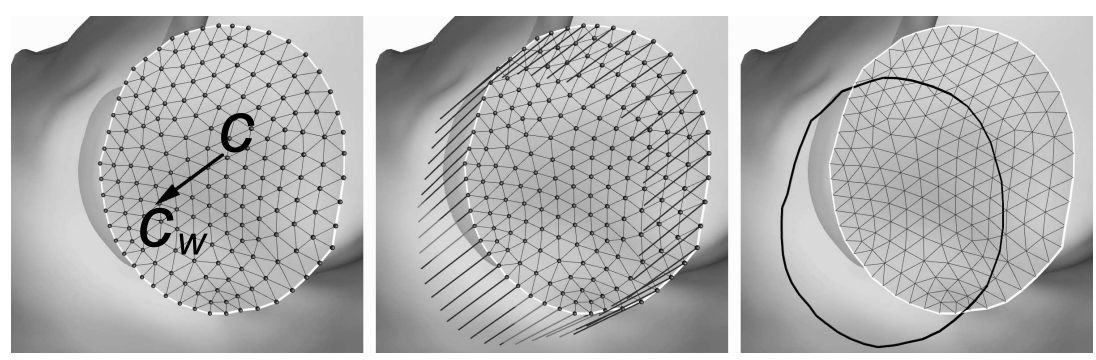

scalar values are more likely to lie on an attachment site. This assumption and the planar character of the structures of interest allow us to compute the displacement vector $\vec{v}$ as the translation of the geometric center of mass $\vec{c}$ and the weighted center of mass $\overrightarrow{c_{w}}$ on the attachment sites triangle vertices using the nodal scalar values as weights (Fig. 4). In a final step the displacement is applied to the contour, by adding the tangentially projected direction and magnitude of $\vec{v}$ to every contour control point. The resulting $3 \mathrm{D}$ coordinate is then projected back onto the surface, creating the new adapted contour.

To prove the potential of our method we validated the results in two stages: First the outputs of the geometric model fitting were compared to the manual segmentations by calculating a two sided surface distance. This was repeated for the locally adapted attachment sites.

\section{$3 \quad$ Results}

The average surface distance measures after the statistical shape fitting are presented in table 1. The maximum distances varied in a range from $1.25 \mathrm{~mm}$ (ACL) to $13.79 \mathrm{~mm}$ (LCL). The dimension of the attachment sites lay between $10 \mathrm{~mm}$ to $35 \mathrm{~mm}$. All the geometrical reconstructed insertion sites were at least partly overlapping with their corresponding manual segmentations (surface projections), which is an important precondition for the following local adaptation. Comparing the results before and after image based adaptation from table 1 exposes no significant changes. In some cases the adaptation step even lead to an increase in the measured surface distances. Especially the ACL was prone to misalignment, due to the problems mentioned in section 2.2. For the PCL the results after image based adaptation improved in all data sets.

\section{Discussion}

We presented a method to segment bony structures including surface representations of ligament attachment sites from unknown CT data sets. This is done 
Table 1. Average surface distances of reconstructed attachment sites to manually segmented attachment sites before and after image based adaptation (in $\mathrm{mm}$ )

\begin{tabular}{lllllll}
\hline Name & Stage & Mean & Deviation & RMS & Max & Median \\
\hline PCL & before & 0.797 & 0.756 & 1.120 & 3.315 & 0.420 \\
& after & 0.750 & 0.689 & 1.040 & 3.045 & 0.404 \\
ACL & before & 1.055 & 1.034 & 1.488 & 4.223 & 0.650 \\
& after & 1.099 & 1.013 & 1.511 & 4.149 & 0.729 \\
\multirow{2}{*}{ LCL } & before & 1.018 & 1.176 & 1.560 & 5.091 & 0.592 \\
& after & 1.069 & 1.253 & 1.650 & 5.122 & 0.550 \\
MCL & before & 0.494 & 0.658 & 0.828 & 3.456 & 0.236 \\
& after & 0.548 & 0.697 & 0.891 & 3.389 & 0.256 \\
\hline
\end{tabular}

by adjusting a geometric model and performing an image based adaptation on the surface of the extracted model.

The results show that low-level methods, like our adaptation step, are not sufficient for attachment site reconstruction in CT data. The reasons can be found in the data itself (the weak soft tissue contrast) and the basic character of our displacement strategy. To overcome the first problem we are going to apply our method to other image modalities with higher soft tissue contrast like MRI. The improvement of the method itself will be our primary objective. This includes a more sophisticated gray value analysis and a more complex representation of the attachment site (e.g. a 3D model of the ligaments), to include more model knowledge into the reconstruction process. However, looking at the results for the PCL, there is a potential and a demand for an improved image based adaption step. Even if this step fails, we could show that the fitting of the geometric model provides a good guess for the location of the attachment sites.

All the results presented in this work are subject to a bigger clinical study with more data sets and exact knowledge of the patient's attachment site extracted from specialized MRI sequences or Image-Guided Surgery systems.

Acknowledgement. This work is partly funded by the European Commission (FP6 IST Project DeSSOS (027252) and the DFG Research Center MatheON.

\section{References}

1. Blemker SS, Asakawa DS, Gold GE, et al. Image-based musculoskeletal modeling: Applications, advances, and future opportunities. J Magn Reson Imaging. $2007 ; 25(2): 441-51$.

2. Kaptein BL, van der Helm FCT. Estimating muscle attachment contours by transforming geometrical bone models. J Biomech. 2004 Mar;37(3):263-73.

3. Lamecker H, Seebaß M, Hege HC, et al. A 3D statistical shape model of the pelvic bone for segmentation. Proc SPIE. 2004;5370:1341-51.

4. Kainmüller D, Lange T, Lamecker H. Shape constrained automatic aegmentation of the liver based on a heuristic intensity model. Proc MICCAI Workshop 3D Segmentation in the Clinic: A Grand Challenge. 2007; p. 109-16. 